\title{
Álgebras Booleanas Super-Magras y Algunas Aplicaciones a la Teoría de Modelos
}

JAIME POSADA

japosada@poligran.edu.co

\section{RESUMEN}

En este trabajo es un acercamiento a las álgebras booleanas super-magras que permiten estudiar facetas de la teoría de modelos presentes en la demostración del teorema de Morley. Esencialmente son álgebras booleanas tales que su espacio de Stone no crece demasiado. Su estudio permitirá simplificar algunos argumentos involucrados en el teorema de Morley, entre ellos la siguiente implicación:

Si $T$ es una teoría $\omega$-estable enumerable con modelos infinitos entonces $T$ es $\kappa$-estable para todo $\kappa \geq \omega$.

\section{ABSTRACT}

In this article we work with certain boolean algebras called super-lean which can be used to study some modeltheoretic facts involved in the proof of Morley's theorem. A super-lean algebra is essentially an algebra such that its Stone space does not grow too much in size. Using those algebras, one can simplify some arguments involved in Morley's theorem such as the following implication: If

$T$ is a $\omega$-stable countable theory with infinite models, then $T$ is $\omega$-stable for all $\kappa \geq \omega$.

PALABRAS CLAVE/KEYWORDS

Algebras Booleanas, Teoría de Modelos / Boolean Algebras, Model Theory

\section{Jaime Posada}

Se desempeña como profesor de tiempo completo del departamento de matemáticas del Politécnico Grancolombiano desde el segundo semestre de 2006. Cuenta con un M.A en matemáticas finalizado en mayo de 2006 en la Univeridad de Wisconsin en Madison.

\section{Introducción}

Łos en los años 50 conjeturó que si una teoría $T$ enumerable es categórica en algún cardinal no enumerable entonces $T$ es categórica en todos los cardinales no enumerables. Morley probó esta conjetura en el 65 para teorías enumerables [M65] y en ese camino surgió la noción de estabilidad.

Para ello introdujo espacios topológicos de tipos, definiendo un rango (hoy en día conocido como rango de Morley) en fórmulas y tipos. La construcción de este rango se basa en el rango de Cantor-Bendixson para espacios topoló- gicos. El análisis del rango permite calcular los tamaños de los espacios de tipos. Una teoría se dice totalmente trascendente si todos los tipos tienen rango de Morley ordinal. Lo anterior es equivalente a que sobre subconjuntos contables, existan a lo más contables tipos; esto último es llamado $\omega$-estabilidad. Este concepto es clave para el desarrollo de la prueba del teorema de Morley; permite construir modelos primos y atómicos sobre subconjuntos potencialmente no enumerables, etcétera.

Łos espacios de tipos son espacios topológicos booleanos y un tipo maximal puede verse como un ultrafiltro de una álgebra de Lindenbaum. Además estas álgebras booleanas permiten estudiar frecuentemente algunas propiedades modelo-teóricas de una teoría de primer orden. Como ejemplo se tiene al teorema de Ryll-Nardzewski: Una teoría enumerable $T$ es $\omega$-categórica si y solamente si para todo $n<\omega, B_{n}(T)$ (álgebras de Lindenbaum) es finita.

En este trabajo se pretenden exponer herramientas booleanas que permiten estudiar facetas de la teoría de modelos presentes en la demostración del teorema de Morley. 
La principal herramienta es el análisis del tamaño del espacio de Stone de una álgebra booleana. Concretamente se estudian las álgebras booleanas que posteriormente se llamarán super-magras. Esencialmente son álgebras booleanas tales que su espacio de Stone no crece demasiado en tamaño. Su estudio permitirá separar algunos argumentos puramente booleanos involucrados en el teorema de Morley, entre ellos la $\kappa$ - estabilidad de una teoría. Al usar las álgebras super-magras, se prueba la equivalencia entre la noción modelo-teorica de $\omega$-estabilidad y el hecho de que ciertas álgebras de Lindenbaum sean super-magras. Como corolario a este hecho se tendrá la $\kappa$-estabilidad $(\kappa \geq \omega$.) de una teoría $\omega$-estable. Los anteriores aspectos, entre otros, permitirán por tanto investigar si el teorema de Morley es un hecho "booleano".

Comenzamos este trabajo con una breve introdu cción al álgebra booleana y a la teoría de modelos. Posteriormente se exponen algunas de las conexiones presentes entre dichas ramas.

\section{Notación y Convenciones}

-Los ordinales y cardinales se notarán indistintamente con letras griegas. Se reservarán las letras $\kappa, \lambda, \mu, \nu$. para cardinales y $\alpha, \beta, \gamma, \delta, \ldots$ para ordinales.

-Las letras caligráficas $\mathcal{A}, \mathcal{B}, \mathcal{C}, \ldots$ denotan álgebras booleanas.

-Las letras góticas $\mathfrak{A}, \mathfrak{B}, \mathfrak{C}$, . denotan estructuras y A,B,C, $\ldots$ sus universos.

-Sea $\mathcal{L}$ un lenguaje de primer orden. Entonces $\mathcal{L}^{n}$ denota el conjunto de fórmulas de primer orden de $\mathcal{L}$ cuyas variables libres están en el conjunto $\left\{x_{i}: i<n\right\}$.

- Si $\mathrm{X}$ es un conjunto, $<\omega X$ es el conjunto de todas las sucesiones finitas de X. Es decir: ${ }^{<\omega} X=\bigcup_{n<\omega} X^{n}$.

\section{2. ÁLGEBRAS BOOLEANAS}

\subsection{Conjuntos parcialmente ordenados}

Definición 2.1. Un orden parcial en un conjunto $P$ es una relación binaria $\leq$ que satisface las siguientes propiedades:
$O P_{1}$. Para todo $x \in P, x \leq x$ (reflexividad).

$O P_{2}$. Para cualesquiera $x, y \in P$, si $x \leq y, y \leq x$, entonces $x=y$ (antisimetría).

$O P_{3}$. Para cualesquiera $x, y \in P$ si $x \leq y, y \leq z$, entonces $x \leq z$ (transitividad).

Un conjunto parcialmente ordenado es un conjunto dotado de un orden parcial. Si $A$ es subconjunto de un conjunto parcialmente ordenado $P$, una cota superior de $A$ es un elemento $z \in P$ tal que para todo $x \in A, x \leq z$ Un elemento $z \in P$ es mínima cota superior de $A$ si es una cota superior de $A$ y para cualquier otra cota superior $w$ de $A, z \leq w$. Si existe la mínima cota superior de $A$ es única por antisimetría y se denota $\sup (A)$. Se define $x \vee y=\sup (\{x, y\})$. Similarmente se define la máxima cota inferior y se denota por $x \wedge y$. Es fácil ver que las operaciones $\vee$ y $\wedge$ son asociativas, conmutativas $\mathrm{e}$ idempotentes (esto último es que $x \vee x=x \wedge x=x$ ).

Un retículo es un conjunto parcialmente ordenado donde cada par de elementos $x, y \in P$ tienen una mínima cota superior y una máxima cota inferior.

Un retículo $L$ con $\mathbf{0}$ y $\mathbf{1}$ es un retículo con elementos $\mathbf{0}, \mathbf{1}$ $\in L,(\mathbf{0} \neq \mathbf{1})$ tales que $\mathbf{0} \leq x \leq$ para todo $x \in P$.

Un retículo $L$ se dice complementado si tiene $\mathbf{0}, \mathbf{1}$ y para cada $x \in L$ existe un elemento $y \in L$, llamado un complemento para $x$, , que satisface:

$$
x \vee y=1 \text { y } x \wedge y=0
$$

Una retículo $L$ se dice distributivo si para todo $x, y, z \in L$ las siguientes identidades son ciertas:

$$
\begin{aligned}
& (x \wedge y) \vee z=(x \vee z) \wedge(y \vee z) \\
& (x \vee y) \wedge z=(x \wedge z) \vee(y \wedge z)
\end{aligned}
$$

Definición 2.2. Una álgebra booleana es un retículo complementado y distributivo.

Se puede probar que en una álgebra booleana el complemento de un elemento es único. De ahora en adelante el complemento de $x$ se notará $x^{*}$.

Definición 2.3. Sean $\mathcal{B}_{1}, \mathcal{B}_{2}$ álgebras booleanas. Un homomorfismo de $\mathcal{B}_{1}$ en $\mathcal{B}_{2}$ es una aplicación $F: \mathcal{B}_{1} \rightarrow \mathcal{B}_{2}$ que respeta la operaciones booleanas. Es decir: Para todo $x, y \in \mathcal{B}_{1}, F(x \wedge y)=F(x) \wedge F(y)$. Similarmente para $\vee,{ }^{*}$. Un isomorfismo de $\mathcal{B}_{1}$ en $\mathcal{B}_{2}$ es un homomorfismo biyectivo. 
Definición 2.4. Una subálgebra de una álgebra booleana $\mathcal{B}$ es un subconjunto no vacío $\mathcal{A}$ que es cerrado bajo las operaciones booleanas en $\mathcal{B}$. Es fácil probar que la intersección de una familia de subálgebras de una álgebra booleana es también una álgebra booleana.

Por lo anterior, dado $\mathrm{X}$ subconjunto de una álgebra booleana $\mathcal{B}$,el conjunto $\langle X\rangle=\bigcap\{\mathcal{A} \subseteq \mathcal{B}: X \subseteq \mathcal{A}$ y $\mathcal{A}$ es subálgebra de $\mathcal{B}\}$ es una subálgebra de $\mathcal{B}$, la cual se llamará subálgebra de $\mathcal{B}$ generada por $X$.

Lema 2.1. $\mathrm{Si} X$ es infinito entonces $|\langle X\rangle|=|X|{ }^{1}$

Demostración: Se puede demostrar que la subálgebra generada por un conjunto $\mathrm{X}$ consiste de los elementos de la forma $\bigvee_{j=1}^{n} \bigwedge_{k=1}^{m} x_{j k}$ donde para todo

$j, k, x_{j k} \dot{\in} X$ o $x_{j k}^{*} \in X$ [BM, CH. $4, \S 3$,

Problem2.4,(ii)].Dèla anterior construcción finitaria se sigue que $|\langle X\rangle|=|X y|$ a que si $S_{\omega}(X)$ denota el conjunto de las partes finitas de $X$, entonces si $X$ es infinito tenemos que $\left|S_{\omega}(X)\right|=|X|$. $\forall$

\subsection{Filtros y Ultrafiltros}

Definición 2.5. Un filtro propio en una álgebra booleana $\mathcal{B}$ es un subconjunto no vacío $F$ de $\mathcal{B}$ que satisface las siguientes condiciones:

$F_{1}$. Para todo $x, y \in F, x \wedge y \in F$.

$F_{2}$. Para todo $x \in F, y \in \mathcal{B}$, si $x \leq y$ entonces $y \in F$.

$F_{3} . \mathbf{0} \notin F$.

De ahora en adelante, al referirnos a un filtro, nos estaremos refiriendo a un filtro propio.

Ejemplos:

1. $\{1\}$ es un filtro.

2. Para cada $x \in \mathcal{B}$ el conjunto $F_{x}=\{y \in \mathcal{B}: x \leq y\}$ es un filtro, llamado el filtro principal generado por $x$.Es sencillo probar que si $\mathcal{B}$ es finita entonces todo filtro en $\mathcal{B}$ es principal.

Definición 2.6. Un subconjunto $X$ de una álgebra booleana $\mathcal{B}$ se dice que tiene la propiedad de intersecciones finitas (abreviada p.i.f) si cuando $x_{1}, x_{2}, \ldots, x_{k} \in X$ entonces $x_{1} \wedge x_{2} \wedge \ldots \wedge x_{k} \neq 0$.

$\mathrm{La}$ anterior propiedad justamente indica cuando un subconjunto de una álgebra booleana esá incluido en un filtro, para ello:

1Este lema es válido en cualquier álgebra con operaciones finitarias.
Teorema 2.1. Un subconjunto $X$ de un álgebra booleana $\mathcal{B}$ está incluido en algún filtro en $\mathcal{B}$ si y solo si $\mid X$ tiene la p.i.f.

Demostración: Ver [BS, CH. 1, § 2, Lemma 2.8].

Los filtros de un álgebra están parcialmente ordenados por inclusión. Los filtros que son maximales respecto a este orden se les llama ultrafiltros. Los ultrafiltros se pueden caracterizar como sigue.

Teorema 2.2.Si $F$ es un filtro en una álgebra booleana $\mathcal{B}$, $F$ es ultrafiltro si y solo si para cada $x \in \mathcal{B}, x \in F o x^{*} \in F$, pero no ambos.

Demostración: Ver [BS, CH. 1, § 3, Lemma 3.1]. $\checkmark$

Surge la pregunta si dada una álgebra booleana $\mathcal{B}$ realmente existe un ultrafiltro $U$ en $\mathcal{B}$. La respuesta es afirmativa asumiendo el axioma de elección como se indica en el siguiente teorema.

Teorema 2.3. (TEOREMA DEL ULTRAFILTRO). Todo filtro en una álgebra booleana puede extenderse a un ultrafiltro.

Demostración: Se sigue de un uso estándar del Lema de Zorn.

Corolario 2.1. Un subconjunto de una álgebra booleana está incluido en un ultrafiltro si y solo si cumple la p.i.f.

Demostración: Es una consecuencia del teorema 2.1 y del teorema del ultrafiltro.

\subsection{Dualidad de Stone}

En los artículos [St36], [St37], M.H. Stone explora la aplicación de conceptos topológicos a la teoría de álgebras booleanas y las aplicaciones de la teoría de anillos booleanos a la topología general. En esta sección exponemos algunos de los resultados de M.H. Stone.

Definición 2.7. Un espacio topológico $(X, \tau)$ es un espacio Booleano si es (i) de Hausdorff, (ii) es compacto y (iii) tiene una base de clopens. 
Definición 2.8. Sea $\mathcal{B}$ una álegebra booleana. Definimos $S(\mathcal{B})$ como el espacio topológico que consta de la cole cción de todos los ultrafiltros de $\mathcal{B}$ y cuya topología está dada por la base que consiste de todos los conjuntos $K_{x}=\{U \in S(\mathcal{B}): x \in U\}$ para $x \in \mathcal{B}$.El espacio $S(\mathcal{B})$ se llamará espacio de Stone de $\mathcal{B}$.

Teorema 2.4. (Stone). El espacio de Stone $S(\mathcal{B})$ de una álgebra booleana $\mathcal{B}$ es un espacio booleano y la aplicación $\varphi: \mathcal{B} \rightarrow C(S(\mathcal{B}))$ tal que $\quad \varphi(x)=K_{x} \quad$ es un isomorfismo de $\mathcal{B}$ en $\mathcal{C}(S(\mathcal{B}))$.

Corolario 2.2. (Teorema de Representación de Stone). Toda álgebra booleana $\mathcal{B}$ es isomorfa a una subálgebra $\operatorname{de} \mathbb{P}(X)$ para $X$ algún conjunto.

Teorema 2.5. (Stone). Cada espacio booleano es homeomorfo al espacio de Stone de su álgebra característica.

Los anteriores teoremas establecen una dualidad en el sentido que ' $S$ ' y ' $C$ ' son operaciones 'inversas' y por lo tanto hay una correspondencia uno a uno entre 'algebras booleanas y espacios booleanos. De hecho M.H Stone establece las siguientes correspondencias (entre otras):

$$
\begin{aligned}
\text { Álgebras booleanas } & \leftrightarrow \text { Espacios booleanos } \\
\text { Filtros } & \leftrightarrow \text { Subconjuntos cerrados } \\
\text { Homomorfismos } & \leftrightarrow \text { Funciones continuas }
\end{aligned}
$$

Para una demostración de los anteriores resultados remitimos al lector a [BS, CH. 1, § 6].

\section{Cardinalidad de espacios de Stone 3.1. Cotas superior e inferior}

Sea $\mathcal{B}$ una álgebra booleana finita. Se puede probar que $|\mathcal{B}|=2^{|S(\mathcal{B})|}$ y por lo tanto $|S(\mathcal{B})|<|\mathcal{B}|$. La situación es completamente distinta cuando $\mathcal{B}$ una álgebra booleana infinita como se mostrará a continuación.

Lema 3.1. Sea $A$ subálgebra propia de una álgebra booleana $\mathcal{B}$ y sea $u \in \mathcal{B} \backslash \mathcal{A}$. Entonces existen ultrafiltros $F, G \in S(\mathcal{B})$ tales que $u \in F \backslash G$ y $F \cap \mathcal{A}=G \cap \mathcal{A}$.
Demostración: Sea $\varphi: \mathcal{B} \rightarrow C(S(\mathcal{B}))$ isomorfismo como en el teorema de representación de Stone. Se demostrará en realidad que existen $F^{\prime} \in \varphi(u), G^{\prime} \in \varphi\left(u^{*}\right)$ tales que para todo $a \in \mathcal{A}, H^{\prime \prime} \in \varphi(a) \Leftrightarrow G^{\prime} \in \varphi(a) ;$ y con ello se sigue que $u \in F^{\prime \prime} \backslash G^{\prime}$ y $H^{\prime \prime} \cap \mathcal{A}=G^{\prime} \cap \mathcal{A}$ ya que si $x \in F^{\prime} \cap \mathcal{A}$,tenemos que $x \in F^{\prime} \cap \mathcal{A}$. y por lo tanto $G^{\prime} \in \varphi(x)$, es decir $x \in G^{\prime} \cap \mathcal{A}$ Similarmente si $x \in F^{\prime} \cap \mathcal{A}$. entonces $x \in F^{\prime} \cap \mathcal{A}$.Supongamos por contradicción que no es posible encontrar dichos ultrafiltros $F^{\prime}, G^{\prime}$, es decir: para todo $F \in \varphi(u)$ y para todo $G \in \varphi\left(u^{*}\right)$,existe $x \in \mathcal{A}$ tal que $F \in \varphi(x)$ y $G \notin \varphi(x)$ o $F \notin \varphi(x)$ y $G \in \varphi(x)$.

Sea $G \in \varphi\left(u^{*}\right)$ fijo, sin pérdida de generalidad para todo $F \in \varphi(u)$, existe $x_{F} \in \mathcal{A}$ tal que $F \in \varphi\left(x_{F}\right)$ y $G \notin \varphi\left(x_{F}\right.$ Se sigue que $\left\{\varphi\left(x_{F}\right): F \in \varphi(u)\right\}$ es un recubrimiento por abiertos de $\varphi(u)$. Como $S(\mathcal{B}$ ) es compacto y $\varphi(u)$.es cerrado entonces $\varphi(u)$.es compacto. Luego existen $x_{F_{1}}, x_{F_{2}}, \ldots, x_{F_{k}} \in \mathcal{A}$ tales que:

$$
\varphi(u) \subseteq \bigcup_{i=1}^{k} \varphi\left(x_{F_{i}}\right) \quad \text { y } \quad G \notin \bigcup_{i=1}^{k} \varphi\left(x_{F_{i}}\right)
$$

como $\varphi$ es isomorfismo

$$
\varphi(u) \subseteq \varphi\left(\bigvee_{i=1}^{k} x_{F_{i}}\right) \quad \text { y } \quad G \notin \varphi\left(\bigvee_{i=1}^{k} x_{F_{i}}\right)
$$

como $A$ es una subálgebra de $\mathcal{B}$ se sigue que para todo $G \in \varphi\left(u^{*}\right)$, existe $x_{G}=\bigvee_{i=1}^{k} x_{F_{i}} \in \mathcal{A}$ tal que $\varphi(u) \subseteq \varphi\left(x_{G}\right)$ y $G \notin \varphi\left(x_{G}\right)$. Se sigue que $\left\{\varphi\left(x_{G}^{*}\right): G \in \varphi\left(u^{*}\right)\right\}$ es un recubrimiento por abiertos de $\varphi\left(u^{*}\right)$. Por compacidad de nuevo, existen $x_{G_{1}}^{*}, x_{G_{2}}^{*}, \ldots, x_{G_{n}}^{*} \in \mathcal{A}$ tales que:

$$
\varphi\left(u^{*}\right) \subseteq \bigcup_{i=1}^{n} \varphi\left(x_{G_{i}}^{*}\right) \quad \text { y } \quad \forall i \quad \varphi(u) \subseteq \varphi\left(x_{G_{i}}\right)
$$

tomando complementos tenemos:

$$
\bigcap_{i=1}^{n} \varphi\left(x_{G_{i}}^{*}\right)^{c} \subseteq \varphi(u)
$$


o equivalentemente:

$$
\varphi\left(\bigwedge_{i=1}^{n} x_{G_{i}}\right) \subseteq \varphi(u)
$$

por otro lado como $\varphi(u) \subseteq \varphi\left(x_{G_{i}}\right)$ para toda $i$, entonces

$$
\varphi(u) \subseteq \varphi\left(\bigwedge_{i=1}^{n} x_{G_{i}}\right)
$$

concluimos de lo anterior que

$$
\varphi(u)=\varphi\left(\bigwedge_{i=1}^{n} x_{G_{i}}\right)
$$

Como $\varphi$ es isomorfismo y $A$ es subálgebra de $\mathcal{B}$ tenemos que

$$
u=\bigwedge_{i=1}^{n} x_{G_{i}} \in \mathcal{A}
$$

pero esto es una contradicción ya que habíamos supuesto $u \in \mathcal{B} \backslash \mathcal{A}$.

Teorema 3.1. Sea $\mathcal{B}$ una álgebra booleana infinita. Entonces $|\mathcal{B}| \leq|S(\mathcal{B})| \leq 2^{|\mathcal{B}|}$

Demostración: Sea $|\mathcal{B}|=\kappa \mathrm{y}$ supongamos por contradi cción que $|S(\mathcal{B})|<\kappa$. Sea

$$
X=\{(F, G) \in S(\mathcal{B}) \times S(\mathcal{B}): F \backslash G \neq \emptyset\}
$$

Para cada $\quad x=(F, G) \in X$, sea $u_{x} \in F \backslash G$. Sea $\Gamma=\left\{u_{x}: x \in X\right\}$. Es fácil ver que para cualquier álgebra booleana $\mathcal{B},|\mathcal{B}| \leq 2^{|S(B)|} \mid$ luego si $|\mathcal{B}|$ es infinita entonces $S(\mathcal{B})$ es infinito.

Por lo tanto $\Gamma$ es infinito. Consideremos $\mathcal{A}=\langle\Gamma\rangle$ (subálgebra generada por $\Gamma$ ) y notemos que:

$$
|\mathcal{A}|=|\Gamma| \leq|S(\mathcal{B}) \times S(\mathcal{B})|=|S(\mathcal{B})|<\kappa
$$

Entonces $|\mathcal{A}|<\kappa$. Ya que $|\mathcal{B}|=\kappa \quad$ es posible encontar $u \in \mathcal{B} \backslash \mathcal{A}$.Por el lema anterior sean $F^{\prime}, G^{\prime} \in S(\mathcal{B})$ tales que $u \in F^{\prime}, u \notin G^{\prime}, F^{\prime} \cap \mathcal{A}=G^{\prime} \cap \mathcal{A}$. Es claro que $x^{\prime}=\left(F^{\prime}, G^{\prime}\right) \in X$ y por lo tanto $u_{x^{\prime}} \in F^{\prime}$, $u_{x^{\prime}} \notin G^{\prime}$. Por construcción $\quad u_{x^{\prime}} \in \mathcal{A} \quad$ y por lo tanto $u_{x^{\prime}} \in F^{\prime} \cap \mathcal{A}$,pero $u_{x^{\prime}} \notin G^{\prime} \cap \mathcal{A}$. Y esto es una contradicción con $F^{\prime} \cap \mathcal{A}=G^{\prime} \cap \mathcal{A}$. Por lo tanto $\kappa \leq|S(\mathcal{B})|$. La desigualdad $|S(\mathcal{B})| \leq 2^{\kappa}$ es inmediata ya que un ultrafiltro en $\mathcal{B}$ es en particular un subconjunto de $\mathcal{B}$.

Observación 3.1. Las anteriores cotas no son estrictas. Por ejemplo: $\mathrm{Si} \mathbb{F}(X)$ es el álgebra booleana de los finitos-cofinitos en $X$ con $X$ infinito, entonces $|\mathbb{F}(X)|=$ $=|S(\mathbb{F}(X))|$ Si $\mathbb{P}(X)$ es el álgebra booleana de partes de $X$, con $X$ infinito, entonces un célebre resultado de Tarski (ver [BS, Ch. $6 \S 1$, Th. 1.5]) establece que $|S(\mathbb{P}(X))|=2^{|\mathbb{P}(X)|}$.

\section{2. Álgebras booleanas super-magras}

En esta sección introducimos la principal herramienta para estudiar el contenido booleano y topológico del teorema de Morley. Particularmente la noción de álgebra booleana super-magra, noción que permitirá controlar el tamaño de los espacios de Stone.

Definición 3.1. Sea $\mathcal{B}$ una álgebra booleana infinita. Diremos que $\mathcal{B}$ es magra si $|\mathcal{B}|=|S(\mathcal{B})|$.

Lema 3.2. Sea $\mathcal{B}$ una álgebra booleana infinita; $\varphi: \mathcal{B} \rightarrow$ $C(S(\mathcal{B}))$ el isomorfismo dado por el teorema de representación de Stone; $x \in \mathcal{B}$ tal que $|\varphi(x)|>|\mathcal{B}|$. Entonces existen $\alpha, \beta \in \mathcal{B}$ tales que $\alpha \wedge \beta=0, \alpha \vee \beta=$ $0, \alpha \vee \beta=x$ y $|\varphi(\alpha)|>|\mathcal{B}|,|\varphi(\beta)|>|\mathcal{B}|$.

Demostracin: Demostremos en realidad que existe $\mathrm{y} \in \mathcal{B}$ tal que $|\varphi(x \wedge y)|>|\mathcal{B}|,\left|\varphi\left(x \wedge y^{*}\right)\right|>|\mathcal{B}|$ y con ello $\alpha=x \wedge y, \beta=x \wedge y^{*}$. Dados $U \in S(\mathcal{B})$ y $y \in \mathcal{B}$ diremos que y es $U$ admisible si $U \in \varphi(x \wedge y)$ y $|\varphi(x \wedge y)| \leq|\mathcal{B}|$.Sea $\Gamma=\{U \in S(\mathcal{B}): \exists y \in \mathcal{B}$ tal que y es $U$-admisible $\}, A=\{y \in \mathcal{B}: \exists U \in S(\mathcal{B})$ tal que y es $U$-admisible $\} \subseteq \mathcal{B}$.Notemos que si $U \in \Gamma$, entonces existe y $\in A$ tal que $U \in \varphi(x \wedge y)$ por lo tanto:

$$
\Gamma \subseteq \bigcup_{a \in A} \varphi(x \wedge a)
$$


de lo cual se sigue

$$
|\Gamma| \leq|A| \sup _{a \in A}|\varphi(x \wedge a)| \leq|\mathcal{B}| \cdot|\mathcal{B}|=|\mathcal{B}|<|\varphi(x)|
$$

por lo tanto sean $F, G \in \varphi(x) \backslash \Gamma, F \neq G$. Sea $y \in \mathcal{B}$ tal que $y \in F, y^{*} \in G$. Entonces $|\varphi(x \wedge y)|>\mid \mathcal{B}$ ,$\left|\varphi\left(x \wedge y^{*}\right)\right|>|\mathcal{B}|$ ya que de lo contrario y es $F$-admisible o $y^{*}$ es $G$ - admisible y por lo tanto $F$ o $G$ estarían en $\Gamma$.

Definición 3.2. Sea $\mathcal{B}$ una álgebra booleana. Decimos que $\mathcal{B}$ es super-magra si todas las subálgebras de $\mathcal{B}$ son magras.

Presentamos ahora una caracterización de las álgebras booleanas super-magras.

Teorema 3.2. Sea $\mathcal{C}$ álgebra booleana infinita. Entonces $\mathcal{C}$ es super-magra si y solamente si todas sus subálgebras enumerables son magras.

Demostración: ( $\Leftarrow$ ") Probaremos la contrarrecíproca. Sea $\mathcal{B}$ una subálgebra de $\mathcal{C}$ tal que $\mathcal{B}$ no es magra. Enton$\operatorname{ces}|S(\mathcal{B})|>|\mathcal{B}|$,o de otra forma: $|\varphi(\mathbf{1})|>\quad$. Por el lema anterior podemos definir inductivamente para cada $k \in \omega, f \in 2^{k}$ un punto $X_{f(0) \ldots f(k-1)} \in \mathcal{B}$ tal que:

1. $\left|\varphi\left(X_{f(0) \ldots f(k-1)}\right)\right|>|\mathcal{B}|$

2. $X_{0} \vee X_{1}=1, X_{0} \wedge X_{1}=0$ y en general:

a) $X_{f(0) \ldots f(k-1) 0} \wedge X_{f(0) \ldots f(k-1) 1}=\mathbf{0}$

b) $X_{f(0) \ldots f(k-1) 0} \vee X_{f(0) \ldots f(k-1) 1}=X_{f(0) \ldots f(k-1)}$

Sea $\mathrm{Ak}=\left\{X_{f}: f \in 2^{k}\right\}, A_{\infty}=\bigcup A_{k}$. Es claro que $\left|A_{\infty}\right|=\aleph_{0}$ y por lo tanto si $\Sigma=\left\langle A_{\infty}\right\rangle$ entonces $|\Sigma|=\aleph_{0}$.

Cada rama del anterior árbol binario tiene la p.i.f y por lo tanto puede extenderse a un ultrafiltro en $\Sigma$. Ahora, si $f, g \in 2^{\omega}$ son tales que $f \neq g$, entonces existe un menor $i \in \omega$ tal que $g(i) \neq f(i)$.

Es así como $X_{f(0) \ldots f(i)} \wedge X_{f(0) \ldots f(i)}=\mathbf{0}$ y por lo tan- to los ultrafiltros asociados a $f$ y $g$ son distintos ya que de lo contrario 0 pertenecería a ellos.

Es así como Es así como $|S(\Sigma)| \geq 2^{\aleph_{0}}$, y de hecho $|S(\Sigma)|=2^{\aleph_{0}}$ ya que por el lema $3.1 .1|S(\Sigma)| \leq 2^{|\Sigma|}=$ $2^{\aleph_{0}}$.

Luego $\Sigma$ es una subálgebra enumerable de $\mathcal{B}$ (y por lo tanto de $\mathcal{C}$ ) no magra.

$(" \Rightarrow ")$ Esta dirección es clara.

Corolario 3.1. No existe álgebra booleana $\mathcal{B}$ enumerable tal que

$$
\aleph_{0}<|S(\mathcal{B})|<2^{\aleph_{0}}
$$

Demostración: Se demostrará en realidad que si $\mathcal{B}$ es un álgebra booleana enumerable y $|S(\mathcal{B})|<2^{\aleph_{0}}$, entonces $|S(\mathcal{B})|=\aleph_{0}$, y con ello el tamaño dèl espacio de Stone es enumerable o es el máximo posible.

Supongamos por contradicción que $|\mathcal{B}|=\aleph_{0}$ y $|S(\mathcal{B})|$ $>\aleph_{0}$, es decir: $\mathcal{B}$ no es magra. Entonces por la prueba del teorema anterior existe $\mathcal{A}$ subálgebra enumerable de $\mathcal{B}$ tal que $|S(\mathcal{A})|=2^{\aleph_{0}}$. Ya que la inclusión de $\mathcal{A}$ en $\mathcal{B}$ es un homomorfismo inyectivo entonces

$$
\begin{gathered}
f: S(\mathcal{B}) \rightarrow S(\mathcal{A}) \\
U \mapsto U \cap \mathcal{A}
\end{gathered}
$$

es continua y sobreyectiva (ver [BM, CH. $4, \S 6$, Th 6.4 ]). Por lo tanto $|S(\mathcal{B})| \geq|S(\mathcal{A})|=2^{\aleph_{0}}$. Y esto último contradice que $|S(\mathcal{B})|<2^{\aleph_{0}}$. Por lo tanto $|S(\mathcal{B})|=\aleph_{0}$.

Presentamos ahora otra caracterización de las álgebras booleanas super-magras.

Teorema 3.3. Sea $\mathcal{B}$ una álgebra booleana. $\mathcal{B}$ es supermagra si y solamente si no existe árbol binario

$\left(x_{s}: s \in \epsilon^{<\omega_{2}} 2\right), x_{s} \neq 0$ contenido en $\mathcal{B}$ tal que

$x_{s \frown 0} \wedge x_{s \frown 1}=\mathbf{0}$ y $x_{s \frown 0} \vee x_{s \frown 1}=x_{s}$.

Demostración: Si existe un árbol binario $\left(x_{s}: s \in<^{<\omega_{2}}\right.$ ), $x_{s} \neq \mathbf{0}$ contenido en $\mathcal{B}$ tal que

$x_{s \frown 0} \wedge x_{s \frown 1}=0$ y $x_{s \frown 0} \vee x_{s \frown 1}=x_{s}$ cada rama de dicho árbol tiene la propiedad de intersecciones finitas por construcción y por lo tanto puede extenderse a un ultrafiltro en $\Sigma=\left\langle\left\{x_{s}: s \in{ }^{<\omega} 2\right\}\right\rangle$

Ultrafiltros asociados a ramas distintas son distintos ya que $x_{s \frown 0} \wedge x_{s \frown 1}=\mathbf{0}$. Luego $|S(\Sigma)|>\omega \mathrm{y}|\Sigma|=\omega$. Es decir $\mathcal{B}$ no es super-magra. Recíprocamente si $\mathcal{B}$ no es 
super-magra entonces existe $\Gamma \leq \mathcal{B}$ tal que $|\Gamma|=$

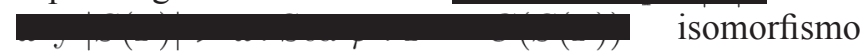
como en el teorema de representación de Stone. Tenemos que $\varphi(\mathbf{1})=S(\Gamma)$ y por lo tanto $|\varphi(\mathbf{1})|>|\Gamma|$ Por el lema 3.2 tenemos que existen $x_{0}, x_{1} \in \Gamma, x_{0}, x_{1} \neq \mathbf{0}$ tale $\mathrm{s}$ que $x_{0} \wedge x_{1}=\mathbf{0}, x_{0} \vee x_{1}=\mathbf{1}$ y $\left|\varphi\left(x_{0}\right)\right|>|\Gamma|$, $\left|\varphi\left(x_{1}\right)\right|>|\Gamma|$.

Iterando este lema obtenemos árbol binario $\left(x_{s}: s \in<\omega_{2}\right), x_{s} \neq \mathbf{0}$ tal que $x_{s \frown 0} \wedge x_{s \frown 1}=\mathbf{0} \mathrm{y}$ $x_{s \frown 0} \vee x_{s \frown 1}=x_{s}$.

\section{Teoría de Modelos}

La teoría de modelos se puede describir como la rama de la lógica matemática que describe las conexiones entre los lenguajes y sus interpretaciones. Los objetos que desempeñan un papel fundamental son las fórmulas de un lenguaje y las estructuras de un lenguaje.

\subsection{Definiciones básicas y algunos resultados}

Presentamos en esta sección algunos hechos y definiciones de la teoría de modelos. Asumimos familiaridad del lector con las definiciones de lenguaje de primer orden, fórmulas, sentencias, estructuras, modelos, satisfacción, consistencia, compacidad. Como referencia de esta sección sitamos a Chang y Keisler en Model Theory (Amsterdam: Noth Holland, 1973).

Definición 4.1. (Definiciones Básicas)

1. Una teoría en un lenguaje $\mathcal{L}$ es un conjunto consistente de sentencias de $\mathcal{L}$. Una teoría es completa si todos los modelos de la teoría satisfacen exactamente las mismas sentencias.

2. Si $\mathcal{L}$ es un lenguaje y $X$ es un conjunto, $\mathcal{L}_{X}$ denota el lenguaje obtenido al añadir a un nuevo símbolo de constante por cada elemento de $X$. Usualmente se notará de igual forma el elemento de $X$ y su símbolo de constante. Dada una estructura $\mathfrak{A}$ y $X \subseteq A, \mathfrak{A}_{X}$ denota la expansión de $\mathfrak{A} a \mathcal{L}_{X}$ en la cual se interpreta al símbolo de constante $a \in X$ por el elemento $a$.

3. Dados dos modelos $\mathfrak{A}$ y $\mathfrak{B}$ en un lenguaje $\mathcal{L}$, una función $F: A \rightarrow B$ es un isomorfismo si $F$ es unā biyección, y para todos los símbolos $S \in \mathcal{L}$ se tiene que $F\left(S^{\mathfrak{A}}\right)=S^{\mathfrak{B}}$

4. La teoría completa de un modelo $\mathfrak{A}$ es el conjunto $T h(\mathfrak{A})=\{\varphi \in \operatorname{Sentencias}(\mathcal{L}): \mathfrak{A} \models \varphi\}$.
5. Dos modelos $\mathfrak{A}$ y $\mathfrak{B}$ son elementalmente equivalentes, denotado $\mathfrak{A} \equiv \mathfrak{B}$, si $T h(\mathfrak{A})=T h(\mathfrak{B})$.

6. Dados dos modelos $\mathfrak{A}$ y $\mathfrak{B}$ en un lenguaje $\mathcal{L}, \mathfrak{A}$ es submodelo de $\mathfrak{B}$, denotado $\mathfrak{A} \subseteq \mathfrak{B}$, si:

$S_{1} . A \subseteq B$

$S_{2} . c^{\mathfrak{A}}=c^{\mathfrak{B}}$ para todo símbolo de constante $c$ en $\mathcal{L}$.

$S_{3} . F^{\mathfrak{A}}=F^{\mathfrak{B}}\left\lceil A^{n}\right.$ para todo símbolo de función $F$ n-ádico en $\mathcal{L}$.

$S_{4} \cdot R^{\mathfrak{A}}=R^{\mathfrak{B}} \cap A^{n}$ para todo símbolo de relación $R$ n-ádico en $\mathcal{L}$.

7. Dados dos modelos $\mathfrak{A}$ y $\mathfrak{B}$ en un lenguaje $\mathcal{L}, \mathfrak{A}$ es submodelo elemental de $\mathfrak{B}$, denotado

$$
\mathfrak{A} \preceq \mathfrak{B} \text {, si: }
$$

$S E_{1} . \mathfrak{A} \subseteq \mathfrak{B}$

$S E_{2}$. Para toda fórmula $\varphi\left(x_{1}, x_{2}, \ldots, x_{n}\right)$

de $\mathcal{L}$, y $a_{1}, a_{2}, \ldots, a_{n} \in A$,

$\mathfrak{A} \models \varphi\left(a_{1}, a_{2}, \ldots, a_{n}\right) \Leftrightarrow \mathfrak{B} \models \varphi\left(a_{1}, a_{2}, \ldots, a_{n}\right)$

Esta última noción trata de capturar "todas" las propiedades de primer orden y no simplemente las propiedades atómicas de un estructura. Por ejemplo: $\mathrm{Si} \mathbb{Q}, \overline{\mathbb{Q}}$ denota el campo de los racionales y el de los algebraicos respectivamente, tenemos que $\mathbb{Q} \subseteq \overline{\mathbb{Q}}$,pero $\mathbb{Q} \npreceq \overline{\mathbb{Q}}$, ya que -1 tiene raíz cuadrada en $\overline{\mathbb{Q}}$, pero no en $\mathbb{Q}$.

\section{Observación 4.1.}

$$
\mathfrak{A} \preceq \mathfrak{B} \text { si y solamente si } \mathfrak{A} \subseteq \mathfrak{B} \text { y } \mathfrak{A}_{A} \equiv \mathfrak{B}_{A} .
$$

El siguiente teorema indica cuando un submodelo puede no ser submodelo elemental: la falta de testigos para los cuantificadores existenciales.

Teorema 4.1. (TEST DE TARSKI-VAUGHT) Dados dos modelos $\mathfrak{A}$ y $\mathfrak{B}$ en un lenguaje $\mathcal{L}, \mathfrak{A} \preceq \mathfrak{B}$ si y solamente si:

1. $\mathfrak{A} \subseteq \mathfrak{B}$.

2. Para todas las fórmulas $\varphi\left(x, x_{1}, \ldots, x_{n}\right)$

$\in \mathcal{L}^{n+1}$ y $a_{1}, a_{2}, \ldots, a_{n}$ en $A$, si $\mathfrak{B} \models \exists x \varphi\left(x, a_{1}, \ldots, a_{n}\right)$, ${ }^{\mathfrak{B}}$. entonces existe $a \in A$ tal que $\mathfrak{B} \models \varphi\left(a, a_{1}, \ldots, a_{n}\right)$. 
Los siguientes teoremas permiten construir modelos con algún tamaño específico. Esta versatilidad muestra algunas de las limitaciones de expresión de la lógica de primer orden.

Teorema 4.2. (TEOREMA DE LOWENHEIM-SKOLEM) Una teoría $T$ en un lenguaje $\mathcal{L}$ que tiene modelos infinitos tiene modelos en cada cardinal $\kappa \geq|\mathcal{L}|+\omega$.

Teorema 4.3. (Teorema descendente de Lowenheim-Skolem-Tarski) Sea $\mathfrak{A}$ un modelo de tipo $\mathcal{L}$. de tamaño $\alpha, y|\mathcal{L}|+\omega \leq \beta \leq \alpha$. Dado $X \subseteq A,|X| \leq \beta$, existe $\mathfrak{C} \preceq \mathfrak{A}$ tal que $X \subseteq C$ y $|C|=\beta$.

\subsection{Saturación y Tipos}

Sea $T$ una teoría consistente en un lenguaje $\mathcal{L}$. Diremos que un conjunto de fórmulas $\Sigma(x) \subseteq \mathcal{L}^{1}$ es consistente con $T$ si $T \cup \Sigma(x)$ es consistente. Un n-tipo parcial de $T$ es un conjunto $\Sigma\left(x_{1}, x_{2}, \ldots, x_{n}\right) \subseteq \mathcal{L}^{n}$ consistente con $T$. Un n-tipo completo de $T$ es un conjunto $\Sigma\left(x_{1}, x_{2}, \ldots, x_{n}\right) \subseteq \mathcal{L}^{n}$ consistente con $T$ y que es maximal respecto a la anterior propiedad. El espacio de tipos de $T$. es el conjunto

$$
S_{n}(T)=\{p: p \text { es un } n \text {-tipo completo de } T\}
$$

Sea $\mathfrak{D} \models T$ y $p \in S_{n}(T)$. Una realización de $p \in S_{n}(T)$ es una tupla $\bar{a} \in D^{n}$ tal que $\mathfrak{D} \models p(\bar{a})$.

Para cada n-tupla $\bar{a}$ en un modelo $\mathfrak{D} d e \mathrm{~T}$ el conjunto

$$
t p^{\mathfrak{D}}(\bar{a})=\left\{\varphi(\bar{x}) \in \mathcal{L}^{n}: \mathfrak{D} \models \varphi(\bar{a})\right\}
$$

es un n-tipo completo de Tel cual es llamado tipo de $\bar{a}$ en $\mathfrak{D}$.Por el Teorema de Compacidad se puede demostrar que todos los tipos son de esta forma; es decir:

$$
S_{n}(T)=\left\{t p^{\mathfrak{D}}(\bar{a}): \bar{a} \in D^{n} \text { para algún } \mathfrak{D} \models T\right\}
$$

Enunciamos a continuacón algunos resultados básicos sobre la realización de tipos.

Lema 4.1. Sea $\mathfrak{A}$ una estructura infinita, $y Y \subseteq A$.

1. Si $p \in S_{n}\left(T h\left(\mathfrak{A}_{Y}\right)\right)$, entonces existe $\mathfrak{B} \succ \mathfrak{A}$ tal que $p$ es realizado en $\mathfrak{B}_{Y}$ y $|B|=|A|$.

2. Existe $\mathfrak{B} \succeq \mathfrak{A},|B| \leq|A| \cdot 2^{\text {máx }(\omega,|Y|)}$, tal que todo $p \in S_{n}\left(T h\left(\mathfrak{A}_{Y}\right)\right)$, es realizado en $\mathfrak{B}$.
Demostración: Ver [S, Prop 15.1].

Definición 4.2. Sea $\mathfrak{A}$ una estructura, $y Y \subset A$. $\mathfrak{A}$ es saturado sobre $Y$, si todo $p \in S_{1}\left(T h\left(\mathfrak{A}_{Y}\right)\right)$ es realizado en $\mathfrak{A}_{Y}$. Supongamos $\kappa$ es un cardinal infinito $\mathfrak{A}$ es $\kappa$-sa turado si $\mathfrak{A}$ es saturado sobre todo $Y \subseteq$ Atal que $|Y|^{-}<\kappa$. $\mathfrak{A}$ es saturado si $\mathfrak{A}$ es $|A|$-saturado.

\section{Ejemplos:}

1. Toda estructura finita es $\omega$-saturada.

2. $\langle\mathbb{Q},<\rangle$ es saturado.

3. C (números complejos vistos como cuerpo algebraicamente cerrado de característica 0) es saturado.

Las estructuras saturadas no son muy comunes. Muchos de los resultados de existencia de estructuras saturadas usan hipótesis conjuntistas (frecuentemente $\mathrm{CH}, \mathrm{GCH}$ o existencia de inaccesibles) que pueden generar dificultades. Concluimos esta sección con el siguiente resultado positivo en la anterior dirección:

Teorema 4.4. Sea $\mathfrak{A}$ una estructura infinita. Para cada cardinal infinito $\kappa$ existe una estructura $\kappa$-saturada $\mathfrak{B} \succeq \mathfrak{A}$ tal que $|B| \leq|A|^{\kappa}$.

Demostración: Ver [S, Th 16.4].

$\nabla$

\section{3. Álgebra de Lindenbaum}

Sea $T$ una teoría consistente en un lenguaje $\mathcal{L}$. En esta sección mostraremos que $T$ da lugar a varias álgebras booleanas que pueden ser usadas para investigar la propiedades modelo-teóricas de $T$. Como referencia a esta sección se tiene [BM, Ch. 5 §5]. Definimos una relación $\cong$ en $\mathcal{L}^{n}$ de la siguiente manera:

$$
\varphi \cong \psi \Longleftrightarrow T \vdash \varphi \leftrightarrow \psi
$$

Se tiene entonces que $\cong$ es una relación de equivalencia en $\mathcal{L}^{n}$. Sea $B_{n}(T)={\mathcal{\mathcal { L } ^ { n }}}^{n} \cong$ Se tiene entonces que $B_{n}(T)$ es una álgebra booleana. Las operaciones booleanas en $B_{n}(T)$ están dadas por:

$$
\begin{aligned}
{[\varphi]_{\cong} \wedge[\psi]_{\cong} } & =[\varphi \wedge \psi]_{\cong} \\
{[\psi]_{\cong}^{*} } & =[\neg \psi]_{\cong}
\end{aligned}
$$


De ahora en adelante al álgebra booleana $B_{n}(T)$ se le llamará álgebra de Lindenbaum de $T$.

\subsection{Ultrafiltros de $B_{n}(T)$ y tipos de $T$}

Sea $T$ una teoría completa y sea $\varphi \in \mathcal{L}^{n}$. Definimos $\|\varphi\|$ como el conjunto $\left\{p \in S_{n}(T): \varphi \in p\right\}$.

El siguiente resultado, que se enuncia sin demostración, le da una topología muy agradable a $S_{n}(T)$.

Teorema 4.5. $\left\{\|\varphi\|: \varphi \in \mathcal{L}^{n}\right\}$ es una base para una topología en $S_{n}(T)$. Con esta topología $S_{n}(T)$ es un espacio booleano.

Por el teorema de representación de Stone, se tiene que $S_{n}(T)$ es homeomorfo al espacio de Stone de una álgebra booleana. En este caso dicha álgebra booleana es $B_{n}(T)$. Esto se debe al siguiente hecho:

$$
\varphi \cong \psi \Longleftrightarrow\|\varphi\|=\|\psi\|
$$

De esto se concluye que $B_{n}(T)$.y el álgebra característica de $S_{n}(T)$ son isomorfas y por lo tanto se tiene:

Teorema 4.6. Sea

$$
\begin{aligned}
F: S_{n}(T) & \rightarrow S\left(B_{n}(T)\right) \\
p & \mapsto\{[\varphi] \cong: \varphi \in p\}
\end{aligned}
$$

Entonces $F$ es un homeomorfismo.

Este último hecho permite identificar de forma natural tipos con ultrafiltros. El siguiente lema se usarará en el último capítulo. La demostración puede encontrarse en [BM].

Lema 4.2. Sea $T$ una teoría completa y Uultrafiltro de $B_{n}(T)$. Entonces $\left\{\varphi:[\varphi]_{\cong} \in U\right\}$ es un conjunto de fórmulas maximal consistente con $T$.

\section{Conexiones}

En esta sección se introduce la noción modelo-teórica de teoría $\kappa$-estable. El resultado principal es el teorema 5.1, el cual relaciona la noción de algebra super-magra y de $\omega$-estabilidad de una teoría.

Haciendo uso de este último teorema se pueden simplificar considerablemente muchos aspectos del teorema de Morley.

1Este lema es válido en cualquier álgebra con operaciones finitarias.

\subsection{Teorías k-estables.}

Comenzamos con una definición. De ahora en adelante $T$ es una teoría enumerable con modelos infinitos.

Definición 5.1. (Morley). Sea $T$ una teoría. $T$ es $\kappa$-estable si $\left|S_{1}\left(T h\left(\mathfrak{A}_{A}\right)\right)\right|=\kappa$ cuando $\mathfrak{A}$ es un modelo de $T$ de cardinalidad $\kappa$.

El siguiente lema es un resultado técnico, necesario para generalizar el teorema 5.1 a todas las álgebras de Lindenbaum.

Lema 5.1. Sea $T$ una teoría $\kappa$-estable. Entonces para todo $\mathfrak{A} \models T$ y $n<\omega$ si $|\mathfrak{A}|=\kappa$ entonces

$$
\left|S_{n}\left(T h\left(\mathfrak{A}_{A}\right)\right)\right|=\kappa \text {. }
$$

Demostración: Por inducción: Sea $\mathfrak{A} \models T$ tal que $|\mathfrak{A}|$ $=\kappa$. Para $n=1$ no hay nada que demostrar. Supongamos $n>1 \quad$ y sea $\mathfrak{B} \succ \mathfrak{A}$ tal que $\mathfrak{B}$ realiza todos los tipos de $S_{n+1}(\mathfrak{A})=S_{n+1}\left(T h\left(\mathfrak{A}_{A}\right)\right)$

(Ver el lema 4.1). Supongamos por contradicción que $\left|S_{n+1}(\mathfrak{A})\right| \neq \kappa$. Hay dos casos:

Caso 1. $\left|S_{n+1}(\mathfrak{A})\right|<\kappa$.

En este caso, ya que $\left|S_{n}(\mathfrak{A})\right|<\left|S_{n+1}(\mathfrak{A})\right|$ tenemos que $\left|S_{n}(\mathfrak{A})\right|<\kappa$, lo cual contradice la hipótesis de inducción.

Caso 2. $\left|S_{n+1}(\mathfrak{A})\right|>\kappa$.

Para cada $q \in S_{n+1}(\mathfrak{A})$, sea $\overline{a_{p}}, b_{p}$ en $B^{n}, B$ respectivamente tales que Consideremos para cada $q \in S_{n+1}(\mathfrak{A}), \operatorname{tp}(q)=$ $\left\{\varphi\left(x_{0}, x_{1}, \ldots, x_{n-1}\right) \in \mathcal{L}_{A}: \mathfrak{B}_{A} \models \varphi\left(\overline{a_{p}}\right)\right\}$.

Se sigue que para cada $q \in S_{n+1}(\mathfrak{A}), t p(q) \in S_{n}$ $\left(T h\left(\mathfrak{B}_{A}\right)\right)=S_{n}\left(T h\left(\mathfrak{A}_{A}\right)\right) \quad$ (Esta última igualdad ya que $\mathfrak{B} \succ \mathfrak{A}$ y por lo tanto $\left.T h\left(\mathfrak{B}_{A}\right)=T h\left(\mathfrak{A}_{A}\right)\right)$. Tenemos entonces que $\Gamma=\{t p(q): q \in$ $\left.S_{n+1}(\mathfrak{A})\right\} \subseteq S_{n}(\mathfrak{A})$ Luego, si $|\Gamma|>\kappa$ entonces $\left|S_{n}(\mathfrak{A})\right|$ esto último contradice la hipótesis de inducción.

Luego $|\Gamma| \leq \kappa$.

Ya que $\kappa^{+}$es regular y $\left|S_{n+1}(\mathfrak{A})\right| \geq \kappa^{+}$, existe, $\Omega \subseteq S_{n+1}(\mathfrak{A}),|\Omega|=k^{+}$tal que todos los tipos $t p(q)$, $q \in \Omega$ son iguales ${ }^{2}$. Sea $p \in \Omega$ fijo, y para cada $q \in \Omega$ sea $\Delta_{q}=\left\{\varphi\left(\overline{a_{p}}, x_{n}\right) \in \mathcal{L}_{A \cup\left\{\overline{a_{p}}\right\}}: \varphi\left(x_{0}, x_{1}\right.\right.$, $\left.\left.\ldots, x_{n}\right) \in q\right\}$.Tenemos entonces que $\left\{\Delta_{q}: q \in \Omega\right\}$ 
es una colección de tipos distintos sobre $\operatorname{Th}\left(\mathfrak{B}_{A \cup\left\{\overline{a_{p}}\right\}}\right)$. Luego $\left|S_{1}\left(T h\left(\mathfrak{B}_{A \cup\left\{\overline{a_{p}}\right\}}\right)\right)\right| \geq \kappa^{+}$.

Ya que $\left|A \cup\left\{\overline{a_{p}}\right\}\right|=\kappa \mathrm{y}|B| \geq \kappa$, por el teorema descendente de Lowenheim-Skolem-Tarski, existe $\mathfrak{C} \preceq \mathfrak{B}$ tal que $|C|=\kappa$ y $A \cup\left\{\overline{a_{p}}\right\} \subseteq C$. Notemos ahora que $\operatorname{Th}\left(\mathfrak{C}_{C}\right)=T h\left(\mathfrak{B}_{C}\right) \supseteq \operatorname{Th}\left(\mathfrak{B}_{A \cup\left\{\overline{a_{p}}\right\}}\right)$ y por lo tanto $\left|S_{1}\left(T h\left(\mathfrak{B}_{A \cup\left\{\overline{a_{p}}\right\}}\right)\right)\right| \leq\left|S_{1}(\mathfrak{C})\right|$. Ya que $T$ es $\omega$-estable y $|C|=\kappa$ entonce $\left|S_{1}(\mathfrak{C})\right|=\kappa . \quad$ Tenemos entonces que:

$$
\kappa^{+} \leq\left|S_{1}\left(T h\left(\mathfrak{B}_{A \cup\left\{\overline{p_{p}}\right\}}\right)\right)\right| \leq \kappa
$$

lo cual es una contradicción. Esto concluye la demostración.

\subsection{Teorías $\omega$-estables y álgebras super-ma- gras}

Presentamos en esta sección la principal aplicabilidad de las álgebras super-magras a la teoría de modelos. Usando argumentos desarrollados en anteriores secciones, presentamos una prueba de un primer resultado (teorema 5.1) debido a Morley en su célebre prueba de la conjetura de Łoś [M65].

Teorema 5.1. Sea $T$ una teoría. $T$ es $\omega$-estable si y solo si para todo $\mathfrak{A} \models T, n<\omega$ el álgebra $B_{n}\left(T h\left(\mathfrak{A}_{A}\right)\right)$ es super-magra.

Demostración: ( $\Rightarrow$ ")Por contradicción. Supongamos $T$ es $\omega$-estable y para algún $n<\omega, B_{n}\left(T h\left(\mathfrak{A}_{A}\right)\right)$ no es super-magra. Por el teorema 3.3 existe árbol binario $\left(\left[\psi_{s}\right]_{\cong}\right.$ : $\left.s \in<\omega_{2}\right)$ tal que $\left[\psi_{s} \frown 0\right] \cong \wedge\left[\psi_{s}-1\right] \cong=0 \mathrm{y}$ $\left[\psi_{s \frown 0}\right]_{\cong} \vee\left[\psi_{s-1}\right]_{\cong}=\left[\psi_{s}\right]_{\cong}$. Consideremos ahora el árbol $\left(\psi_{s}: s \in<^{<\omega} 2\right)$. Sea $X$ el conjunto de parámetros de $\mathfrak{A}$ nombrados en alguna fórmula del anterior árbol. Es claro que $|X|=\omega$. Por el teorema descendente de Lowenheim-Skolem-Tarski, existe $\mathfrak{B} \preceq \mathfrak{A}$ tal que $|B|=$ $\omega$ y $X \subseteq B$. Tenemos luego que $\mathfrak{B} \models T$.

Mostremos ahora que $\left|S_{n}\left(T h\left(\mathfrak{B}_{B}\right)\right)\right|>\omega$ con lo cual se llega a una contradicción, ya que por el teorema anterior, al ser $T \omega$-estable, tenemos que $\left|S_{n}\left(T h\left(\mathfrak{B}_{B}\right)\right)\right|=\omega$. Veamos primero que cada rama del árbol $\left(\psi_{s}: s \in<\omega_{2}\right)$ es consistente con $T h\left(\mathfrak{B}_{B}\right)$. Para ello tomemos un subconjunto finito $\Delta$ de una rama. Por construcción $\bigwedge_{\psi \in \Delta}[\psi]_{\cong}$ $\neq 0$.
Luego $\left[\bigwedge_{\psi \in \Delta} \psi\right]_{\simeq} \neq 0$.Es decir: $\operatorname{Th}\left(\mathfrak{A}_{A}\right) \nvdash \neg \bigwedge_{\psi}, \mathfrak{B}_{B}$ $\psi$. Ya que $T h\left(\mathfrak{B}_{B}\right) \subseteq T h\left(\mathfrak{A}_{A}\right)$ tenemos que $\operatorname{Th}\left(\mathfrak{B}_{B}\right)$ $\nvdash \neg \bigwedge_{\psi \in \Delta} \psi$. Luego $T h\left(\mathfrak{B}_{B}\right) \cup\left\{\bigwedge_{\psi \in \Delta} \psi\right\}$ es consistente. Por compacidad cada rama del anterior árbol es consistente con $T h\left(\mathfrak{B}_{B}\right)$ y por lo tanto se puede extender a $p \in S_{n}\left(T h\left(\mathfrak{B}_{B}\right)\right)$. Ya que $\left[\psi_{s}-0\right]_{\cong} \wedge\left[\psi_{s} \frown 1\right]_{\cong}$ $=\mathbf{0}$ entoncês tipos asociados a ramas distintas son distintos. Se sigue que $\left|S_{n}\left(T h\left(\mathfrak{B}_{B}\right)\right)\right| \geq 2^{\omega}>\omega$.

(" $\Leftarrow$ ") Supongamos que existe $\mathfrak{B} \models T$ tal que $|B|=$ $\omega_{-}$y $\left|S_{1}\left(T h\left(\mathfrak{B}_{B}\right)\right)\right|>\omega$. Tenemos que $\mid$ Sentencias $\left(\mathcal{L}_{B}\right)|=| \mathcal{L}| \pm| B \mid+\omega=\omega$.Luego $\left|\operatorname{Th}\left(\mathfrak{B}_{B}\right)\right|=\omega . \quad$ Se sigue que $\left|B_{1}\left(T h\left(\mathfrak{B}_{B}\right)\right)\right| \leq \omega$ y de hecho $\mid B_{1}(T h$ $\left.\left(\mathfrak{B}_{B}\right)\right) \mid=\omega$ ya que de lo contrario si $\left|B_{1}\left(T h\left(\mathfrak{B}_{B}\right)\right)\right|<\omega$ entonces $\left|S\left(B_{1}\left(T h\left(\mathfrak{B}_{B}\right)\right)\right)\right|<\omega$ (Una álgebra booleana finita tiene finitos ultrafiltros); pero esto contradice que $\left|S\left(B_{1}\left(T h\left(\mathfrak{B}_{B}\right)\right)\right)\right|=\left|S_{1}\left(T h\left(\mathfrak{B}_{B}\right)\right)\right|>\omega$. Luego $B_{1}\left(T h\left(\mathfrak{B}_{B}\right)\right)$ es una 'álgebra booleana enumerable con espacio de Stone no enumerable. Luego $B_{1}\left(T h\left(\mathfrak{B}_{B}\right)\right)$ no es super-magra. Esto concluye la demostración.

Corolario 5.1. (MORLEY). Sea $T$ una teoría. Si $T$ es $\omega$ estable entonces $T$ es $\kappa$-estable para todo $\kappa \geq \omega$.

Demostración: Supongamos $T$ no es $\kappa$-estable en algún $\kappa \geq \omega$. Sea $\mathfrak{A} \models T$ tal que $|\mathfrak{A}|=\kappa \mathrm{y}$ $\left|S_{1}\left(T h\left(\mathfrak{A}_{A}\right)\right)\right|>\kappa$. Tenemos que $\left|B_{1}\left(T h\left(\mathfrak{A}_{A}\right)\right)\right|=\kappa$ y $\left|S\left(B_{1}\left(T h\left(\mathfrak{A}_{A}\right)\right)\right)\right|>\kappa . \quad$ Luego $B_{1}\left(T h\left(\mathfrak{A}_{A}\right)\right)$ no es super-magra. Por el teorema anterior $T$ no es $\omega$-estable. 


\section{BIBLIOGRAFIA}

-[BM] J. L. Bell, M. Machover A course in Mathematical Logic. North-Holland, Amsterdam, 1977.

- [BS] J. L. Bell, A. B. Slomson. Models and Ultraproducts, North-Holland, Amsterdam, 1971.

- [B] S. Buechler. Essential Stability Theory. SpringerVerlag, New York, 1996.

- [C] E. Casanovas. Teor'ia de Modelos. Preprint, 2000.

- [CK] C. C. Chang, H. J. Keisler. Model Theory. NorthHolland, Amsterdam, 1973.

-[K78] J. Knight. Prime and Atomic Models. The Journal of Symbolic Logic, Vol 43, Num 3, 385-393.

- [M65] M. Morley. Categoricity in Power. Trans. Amer. Math. Soc, 114, 514-538, 1965.

- [S] G. Sacks. Saturated Model Theory. W. A. Benjamin, Inc. Reading, Massachusetts, 1972.

- [St36] M.H. Stone. The theory of representation for Boolean algebras. Trans. Amer. Math. Soc, 40, 37-111.

-[St37] M.H. Stone. Applications of the theory of Boolean rings to general topology. Trans. Amer. Math.

Soc, 41, 375-381. 